\title{
Pattern II and pattern III MS are entities distinct from pattern I MS: evidence from cerebrospinal fluid analysis
}

S. Jarius ${ }^{1 *}$, F.B. König ${ }^{2}$, I. Metz ${ }^{2}$, K. Ruprecht ${ }^{3}$, F. Paull ${ }^{3,4}$, W. Brück ${ }^{2^{* \dagger}}$ and B. Wildemann ${ }^{1+}$

\begin{abstract}
Background: The diagnosis of multiple sclerosis (MS) is currently based solely on clinical and magnetic resonance imaging features. However, histopathological studies have revealed four different patterns of lesion pathology in patients diagnosed with MS, suggesting that MS may be a pathologically heterogeneous syndrome rather than a single disease entity.

Objective: The aim of this study was to investigate whether patients with pattern I MS differ from patients with pattern II or III MS with regard to cerebrospinal fluid (CSF) findings, especially with reference to intrathecal IgG synthesis, which is found in most patients with MS but is frequently missing in MS mimics such as aquaporin-4-lgGpositive neuromyelitis optica spectrum disorders and myelin oligodendrocyte glycoprotein-lgG-positive encephalomyelitis.
\end{abstract}

Methods: Findings from 68 lumbar punctures in patients who underwent brain biopsy as part of their diagnostic work-up and who could be unequivocally classified as having pattern I, pattern II or pattern III MS were analysed retrospectively.

Results: Oligoclonal bands (OCBs) were present in $88.2 \%$ of samples from pattern I MS patients but in only $27 \%$ of samples from patients with pattern II or pattern III MS ( $P<0.00004)$; moreover, OCBs were present only transiently in some of the latter patients. A polyspecific intrathecal lgG response to measles, rubella and/or varicella zoster virus (so-called MRZ reaction) was previously reported in $60-80 \%$ of MS patients, but was absent in all pattern II or III MS patients tested ( $P<0.00001$ vs. previous cohorts). In contrast, the albumin CSF/serum ratio (QAlb), a marker of blood-CSF barrier function, was more frequently elevated in samples from pattern II and III MS patients $(P<0.002)$. Accordingly, QAlb values and albumin and total protein levels were higher in pattern II and III MS samples than in pattern I MS samples $(P<0.005$, $P<0.009$ and $P<0.006$, respectively).

Conclusions: Patients with pattern II or pattern III MS differ significantly from patients with pattern I MS as well as from previous, histologically non-classified MS cohorts with regard to both intrathecal IgG synthesis and blood-CSF barrier function. Our findings strongly corroborate the notion that pattern II and pattern III MS are entities distinct from pattern I MS.

Keywords: Multiple sclerosis, Histopathology, Pattern I lesions, Pattern II lesions, Pattern III lesions, Cerebrospinal fluid, Oligoclonal bands, Intrathecal lgG synthesis, Blood-CSF barrier dysfunction, Total protein, QlgG, QAlb

\footnotetext{
*Correspondence: sven.jarius@med.uni-heidelberg.de; wbrueck@med.uni-

goettingen.de

${ }^{\dagger}$ Equal contributors

W.B. and B.W. are equally contributing senior authors.

'Molecular Neuroimmunology Group, Department of Neurology, University

of Heidelberg, Heidelberg, Germany

${ }^{2}$ Department of Neuropathology, University of Göttingen, Göttingen,

Germany

Full list of author information is available at the end of the article
} 


\section{Background}

Histopathological studies have demonstrated at least three different lesion patterns in early disease stages from patients diagnosed with multiple sclerosis (MS), termed patterns I, II and III $[1,2]$. Pattern I lesions show $\mathrm{T}$ cell and macrophage infiltration. Pattern II is defined by additional antibody and complement deposition, suggesting a contribution of humoral mechanisms to disease pathology. Pattern III is characterized by distal oligodendrogliopathy with dysregulated myelin protein expression and oligodendrocyte apoptosis, but still occurs on an inflammatory background. A fourth pattern, defined by oligodendrocyte degeneration in the periplaque white matter, has been described in few autopsy cases of primary-progressive MS, but is rare. These findings raise the possibility that MS, a diagnosis currently based mainly on phenotypical, namely clinical and radiological features [3], may in fact be a pathologically heterogeneous syndrome rather than a single disease entity. Importantly, two recent studies demonstrated intraindividual homogeneity and persistence of pattern I, II and III lesions over time [4,5], further corroborating the notion that lesion pathology may rather define pathogenetically distinct entities than reflect stagedependent processes in the development of lesions.

Intrathecal IgG synthesis, as detected qualitatively by isoelectric focusing (IEF) of cerebrospinal fluid (CSF) and serum or quantitatively by calculation of the immunoglobulin CSF/serum ratio (QIgG), is present in 90-98\% of MS patients, usually remains detectable over the entire course of disease and is considered a diagnostic mainstay in MS [6-9].

In this study, we retrospectively compared the CSF profiles of patients who underwent brain biopsy as part of their diagnostic work-up and who could be unequivocally classified as having pattern I, pattern II or pattern III lesions, respectively.

\section{Methods \\ Patients}

Results from 68 routine lumbar punctures in 33 patients with histopathologically confirmed MS $(16 \times$ pattern II, 7 $\times$ pattern III, $10 \times$ pattern I $[1,2])$ were analysed for OCB frequency and OCB patterns; CSF and serum IgG, IgM and IgA; CSF and serum albumin; IgG, IgM and IgA CSF/ serum ratios (QIgG, QIgM, QIgA); albumin CSF/serum ratio (QAlb); intrathecal IgG response to measles (M), rubella (R) and varicella zoster (Z) (MRZ reaction [MRZR]) [10-14]; CSF total protein (TP) and CSF L-lactate levels; and CSF white cell counts and white cell differentiation. All patients had undergone brain biopsy as part of their diagnostic work-up. All biopsies were histopathologically classified at the Department of Neuropathology, University of Göttingen, Germany (WB, IM, FK), as previously described [1, 2]. All patients were classified based on brain lesions; none was classified based on brainstem, spinal cord or optic nerve lesions. All had early active disease and none had primary-progressive disease or pattern IV lesions. The median age at the time of first lumbar puncture was 36 years (range 13-63). The sex ratio (m:f) was 1:1.75. All patients were of Caucasian origin. Available serum samples were retrospectively tested for aquaporin 4 (AQP4)-IgG and myelin oligodendrocyte glycoprotein (MOG)-IgG using a cell-based assay (CBA) employing formalin HEK293 cells transfected with full-length human M1-AQP4 and M23-AQP4 $[15,16]$ or full-length human MOG $[17,18]$, respectively. All analyses were done retrospectively, in no case were brain, blood or CSF specimens obtained for the present study. AQP4-IgG and MOG-IgG testing and retrospective analysis of the patients' CSF results was performed in an anonymized fashion.

\section{Evaluation of the humoral immune response}

Oligoclonal IgG bands (OCBs) were evaluated according to an international consensus [9]: IEF pattern $1=$ no OCBs in CSF or serum; IEF pattern $2=$ CSF-restricted OCBs; IEF pattern $3=$ CSF-restricted OCBs and additional identical bands in CSF and serum (combination of patterns 2 and 4); IEF pattern $4=$ identical OCBs in CSF and serum ('mirror pattern'); and IEF pattern $5=$ monoclonal bands in CSF and serum. Only IEF patterns 2 and 3 indicate intrathecal IgG synthesis. Quantitative expressions of the intrathecal humoral immune response were based on calculation of the CSF/ serum ratios for IgG (QIgG), IgM (QIgM) and IgA $(\mathrm{QIgA})$ with $\mathrm{Q}_{\mathrm{Ig}}=\operatorname{Ig}_{\mathrm{CSF}[\mathrm{mg} / \mathrm{L}]} / \operatorname{Ig}_{\text {serum }[\mathrm{g} / \mathrm{L}]}$. The upper limits of the respective reference ranges, $\mathrm{Q}_{\lim }(\mathrm{IgG})$, $\mathrm{Q}_{\lim }(\operatorname{IgM})$ and $\mathrm{Q}_{\lim }(\operatorname{Ig} \mathrm{A})$, were calculated against QAlb according to Reiber's revised hyperbolic functions [10]. Values for $\mathrm{Q}_{\mathrm{Ig}}>\mathrm{Q}_{\lim }(\mathrm{Ig})$ were considered to indicate intrathecal immunoglobulin synthesis [10]. The fraction (in \%) of intrathecally produced $\mathrm{Ig}\left(\mathrm{Ig}_{\mathrm{IF}}\right)$ and the absolute amount of locally, i.e. intrathecally, produced Ig (IgG $\left.\mathrm{G}_{\text {loc }}\right)$ were calculated according to the following formulas: $\operatorname{Ig}_{\mathrm{IF}[\%]}=\left[\mathrm{Q} I g-\mathrm{Q}_{\lim }(\mathrm{Ig})\right] \times \operatorname{Ig}_{\text {serum }} \times 100$ and $\operatorname{Ig}_{\text {loc }[\mathrm{mg} / \mathrm{L}]}=\left[\mathrm{QIg}-\mathrm{Q}_{\mathrm{lim}}(\mathrm{Ig})\right] \times \operatorname{Ig}_{\text {serum }}$, respectively [10] Antibody indices (AI) were calculated according to Reiber's formula: $\mathrm{AI}=\mathrm{Q}_{\text {spec }} / \mathrm{QIgG}$, or $\mathrm{AI}=\mathrm{Q}_{\text {spec }} / \mathrm{Q}_{\text {lim }}(\mathrm{IgG})$ if $\left(\mathrm{Q}_{\lim }>\mathrm{QIgG}\right)$, with $\mathrm{Q}_{\text {spec }}=\operatorname{IgG}_{\text {spec }}(\mathrm{CSF}) / \operatorname{IgG}_{\text {spec }}$ (serum). Taking into account the relatively small sample size of the pattern I MS subgroup, we also compared the results for pattern II and pattern III MS with data from 'classical' landmark studies on OCB and MRZR findings in (histologically non-classified) MS [14, 19].

\section{Evaluation of blood-CSF barrier function}

The CSF/serum albumin quotient, QAlb $=\operatorname{Alb}_{\mathrm{CSF}[\mathrm{mg} / \mathrm{L}]} /$ $\mathrm{Alb}_{\text {serum }[\mathrm{g} / \mathrm{L}]}$, was used to assess the blood-CSF barrier 
function. As the upper reference limit of QAlb is age dependent, $\mathrm{Q}_{\lim }(\mathrm{Alb})$ was calculated as $4+(a / 15)$, with $a$ representing the patient's age, according to Reiber et al. [20]. Dysfunction of the blood-CSF barrier was defined as QAlb $>$ Q $\lim ($ Alb).

\section{Cytological examination, total CSF protein and L-lactate} A white cell count $>5 / \mu \mathrm{L}$ was classified as 'increased'. An age-dependent upper reference range for CSF L-lactate was applied $(0-15$ years of age, $1.8 \mathrm{mmol} / \mathrm{L} ; 16-50$ years, $2.1 \mathrm{mmol} / \mathrm{L}$; $>50$ years, $2.6 \mathrm{mmol} / \mathrm{L}[21,22])$. As upper reference limit for total CSF protein, $450 \mathrm{mg} / \mathrm{L}$ was used.

\section{Statistics}

Fisher's exact test and Mann-Whitney $U$ test were used to detect differences between groups. Spearman's rho was calculated to test for correlations. $P$ values $<0.05$ were considered statistically significant. Due to the exploratory nature of this study, no corrections for multiple comparisons were performed. Reiber diagrams were generated using the Protein Statistics in CSF analysis V3.0 software (Comed, Soest, Germany). The study was approved by the institutional review boards of the University of Göttingen and the University of Heidelberg. All CSF parameters evaluated in this study are routinely tested in Germany as part of the diagnostic workup of patients with suspected MS in Germany and are recommended by the guidelines of the German Society of Neurology and by the guidelines of the Germany Society of CSF Analysis and Clinical Neurochemistry [21].

\section{Results}

\section{Epidemiology and autoantibody status}

Epidemiological data for all subgroups are given in Table 1. All serum samples available for retrospective testing $(n=13)$ were negative for AQP4-IgG and MOG-IgG [18].

\section{Oligoclonal bands}

CSF-restricted OCB were found in 15/17 (88.2\%) samples from patients with pattern I MS, but were negative in 27/37 (73\%) of samples from patients with pattern II or pattern III MS $(P<0.00004)$ (Fig. 1). Overall, only 7/22 (31.8\%) pattern II and III patients, but 8/10 (80\%) pattern I MS patients, had OCB at least once $(P<0.021)$. Moreover, OCBs were only transiently positive in 2 of the 7 only OCB-positive pattern II and III patients (patient 1, pattern II: lumbar puncture (LP) \#1 positive, LP \#2 negative; patient 2, pattern III: LP \#1 positive, LP \#2 negative). If only persisting OCB are taken into account, only $22.7 \%(5 / 22)$ patients with pattern II or III MS were positive for OCB $(P<0.006)$ (Table 2). Of note, QIgG was negative at the time of OCB determination in the two cases with transient $\mathrm{OCB}$, indicating very low levels of intrathecal $\mathrm{IgG}$ synthesis in these patients. Identical OCB in serum and CSF without additional CSF-restricted bands (the so-called mirror pattern or IEF pattern 4), suggesting possible systemic inflammation, were found in a single patient with pattern II MS but were absent in all other patients. IEF pattern 5, indicating monoclonal gammopathy, was present in a single pattern II MS patient (still detectable in a second sample taken 24 months later) and transiently in one pattern III patient (negative follow-up sample obtained 36 days later). Comparison of the pattern II or pattern III MS patients' data with data from a reference paper on CSF in MS [19] confirmed the marked difference in OCB frequency $(P<0.000001)$ (see Table 9 for details).

\section{$\lg G$, IgM and IgA CSF/serum ratios}

While 7/17 (41.2) samples from patients with pattern I MS showed elevated QIgG levels, only 5/41 (12.2) of samples from patients with pattern II or pattern III MS did so $(P<0.021)$ (Table 2$)$. Intrathecal production of IgM as indicated by elevated QIgM was rare and was found both in patients with pattern I MS (3/9; 33.3\%) and in patients with pattern II MS (3/12; 25\%). QIgA levels were elevated in a few pattern I patients $(2 / 8$; $25 \%$ ), in a single pattern II patient and in none of the pattern III patients $(1 / 17 ; 5.9 \%)$. Data on both the fractions and the absolute amounts of intrathecally produced IgG, IgM and IgA can be found in Tables 2 and 3. Plots of QIgG, QIgA and QIgM, respectively, against QAlb as a measure of blood-CSF barrier function are shown in Fig. 2.

Table 1 Epidemiological findings

\begin{tabular}{|c|c|c|c|c|c|c|}
\hline & Units & Pattern I MS & Patterns II + III MS & Pattern II MS & Pattern III MS & $\mathrm{AQP4}-\mathrm{Ab}+\mathrm{NMO}[48]$ \\
\hline Patients & $N$ & 10 & 23 & 16 & 7 & 89 \\
\hline Samples & $N$ & 19 & 49 & 30 & 19 & 211 \\
\hline Age at first LP & Years & $30(13-47)$ & $36(20-63)$ & $35(20-51)$ & $38(26-63)$ & 39.5 (14-79) \\
\hline Sex ratio & $m: f$ & 1:1 & $1: 2.3$ & $1: 2.2$ & $1: 2.5$ & 1:12.1 \\
\hline
\end{tabular}

$L P$ lumbar puncture, $m$ male, $f$ female

Results in AQP4-lgG-positive NMO as observed in a previous study [48] are given in the last column for comparison. Note the marked difference in the sex ratios between pattern II MS and NMO. Years are given as median and range 


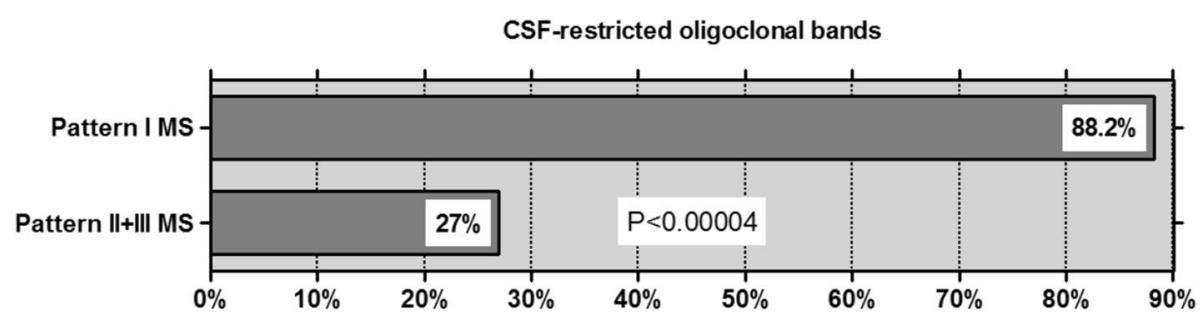

Fig. 1 Frequency of CSF-restricted oligoclonal bands (OCBs) results from 54 LPs in patients with pattern I MS compared to patients with pattern II or pattern III MS. Note that OCBs were present only transiently in 2/7 OCB-positive pattern II/II patients

\section{Immunoglobulin class patterns}

Intrathecal Ig production, if present, was restricted to one immunoglobulin class in most cases (cf. Table 4 for details). A two-class immune reaction, defined as intrathecal production of either IgG and IgM, or IgM and IgA, or IgG and IgA, was present in only four samples (Table 4). None of the samples showed a three-class immune response, defined as combined elevation of QIgG, QIgM and QIgA. Of note, three pattern II patients showed an isolated IgM reaction at least once (patient 1, intrathecal IgM, IgG and IgA fractions, 83, 47 and $0 \%$, respectively, at first lumbar puncture; 73, 0 and $0 \% 10$ days later; and 71, 0 and $0 \% 14$ days later; patient $2,37,0$ and $0 \%$; patient $3,17,0$ and $0 \%$ at first puncture, no intrathecal IgM, IgG and IgA synthesis at repeat puncture at day 34 and day 815 ).

\section{MRZ reaction}

A positive MRZR, as defined by at least two positive IgG-AIs, which has been reported in the literature to be present in around 70\% of patients with MS [10-14, 23], was absent in all samples from patients with pattern II or pattern III MS examined $(P<0.000002$ compared to unselected MS patients [23]). Only two patients with pattern I MS had been tested for MRZR (positive in 1/2, $50 \%$ ), precluding statistical comparisons. A positive AI against at least one of the three constituents (measles virus, rubella virus, varicella zoster virus), which has been reported in the literature to be present in $89 \%$ (158/177) [19] to $94 \%(94 / 100)$ [14] of patients with MS, was absent in $91 \%(10 / 11)$ of samples from pattern II and III patients $(P<0.000001$ vs. references [19] and [14]). MRZR was re-tested in two patients (one with pattern II and one with pattern III MS) later in the disease course and remained negative in both cases. Only a single patient with pattern III MS had a positive AI for measles virus; however, a follow-up examination 1 month later was negative. See Table 5 for details.

\section{Blood-CSF barrier integrity}

A disrupted blood-CSF barrier function was found in only 4/17 (23.5) of samples from patients with pattern I
MS but in 30/43 (69.8) of samples from patients with pattern II or pattern III MS $(P<0.002)$. Accordingly, median QAlb values and median albumin CSF concentrations were higher in pattern II or III samples than in pattern I samples $(P<0.005$ for QAlb, $P<0.009$ for CSF albumin). See Table 6 for details.

\section{Cellular immune response}

CSF pleocytosis was slightly less frequent in pattern II MS samples $(6 / 26 ; 23.1 \%)$ than in pattern I $(5 / 13 ; 38.5 \%)$ and pattern III $(10 / 14 ; 71.4 \%)$ samples $(P<0.02)$. However, median CSF white cell count (WCC) were low in all three subgroups, with only $3 / 49(6.1 \%)$ of samples showing cell counts $>40$ cells $/ \mu \mathrm{L}$ (see Table 7 for details). In patients with pleocytosis, CSF white cells included lymphocytes in all samples examined and monocytes in most, with no significant differences between the groups. Neutrophil granulocytes were present only in five samples from patients with pleocytosis, and the levels were very low $(1 \times$ pattern I MS: 7 cells $/ \mu \mathrm{L}, 5 \%$ neutrophils; 1 $\times$ pattern II MS: 6 cells $/ \mu \mathrm{L}, 3 \%$ neutrophils; $3 \times$ pattern III MS: 17,13 and 7 cells $/ \mu \mathrm{L}, 1-3 \%$ neutrophils) and possibly related to slight blood contamination in one case (722 erythrocytes/ $\mu \mathrm{L})$. Eosinophils were present in only two patients with pleocytosis $(1 \times$ pattern II MS: 69 cells $/ \mu \mathrm{L}, 13 \%$ eosinophils, absent both in a previous sample and in a follow-up sample, AQP4-IgG: negative; $1 \times$ pattern III MS: 7 cells $/ \mu \mathrm{L}, 5 \%$ eosinophils; no erythrocytes in both cases). Sixty-two percent (13/21) of pattern II/III MS samples without pleocytosis showed an elevated QAlb and, thus, an albuminocytological dissociation, but only $12.5 \%(1 / 8)$ of pattern I MS samples ( $\mathrm{P}<0.04$; see Table 7 for details).

\section{Total CSF protein}

Total protein (TP) in the CSF was more frequently elevated in samples from patients with pattern II or III MS samples $(29 / 43 ; 67.4 \%)$ than in pattern I MS (3/15; 20\%) $(P<0.003)$. Accordingly, median TP CSF levels were higher in pattern II or III MS samples (median $550 \mathrm{mg} / \mathrm{dl}$, range 168-1930) than in pattern I MS samples (median 375 , range $187-750)(P<0.006)$, with the highest levels detected in pattern III samples (median 


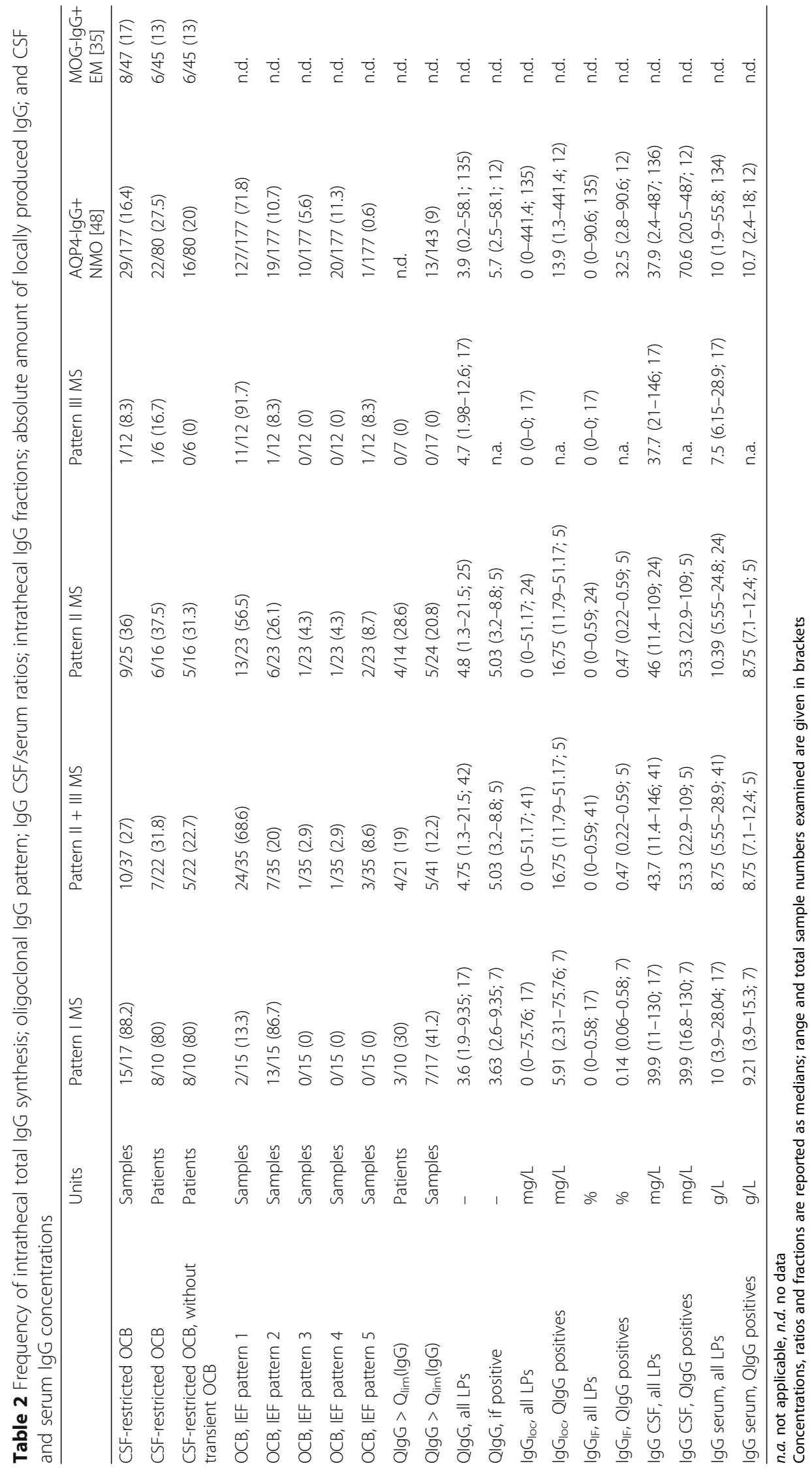




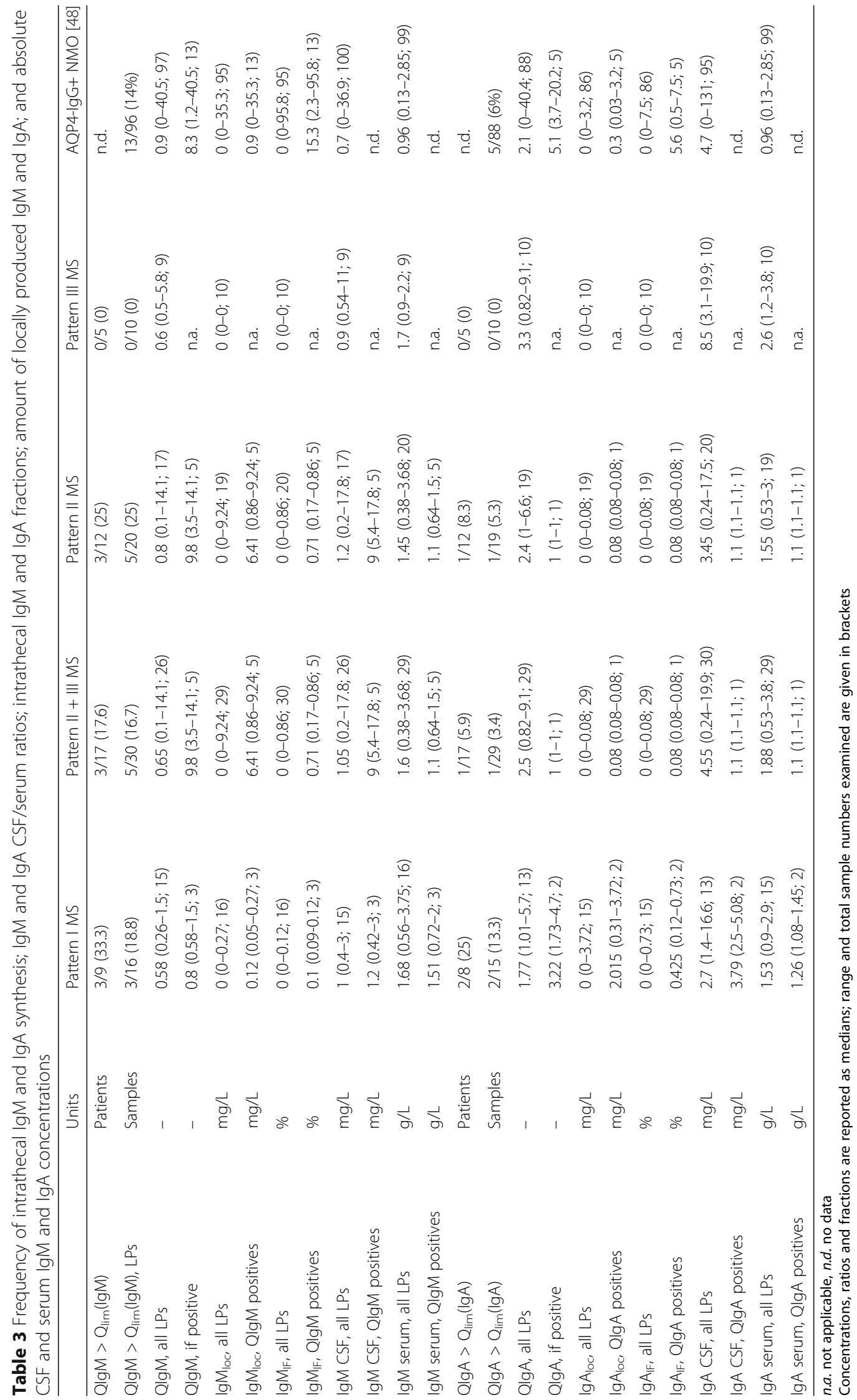




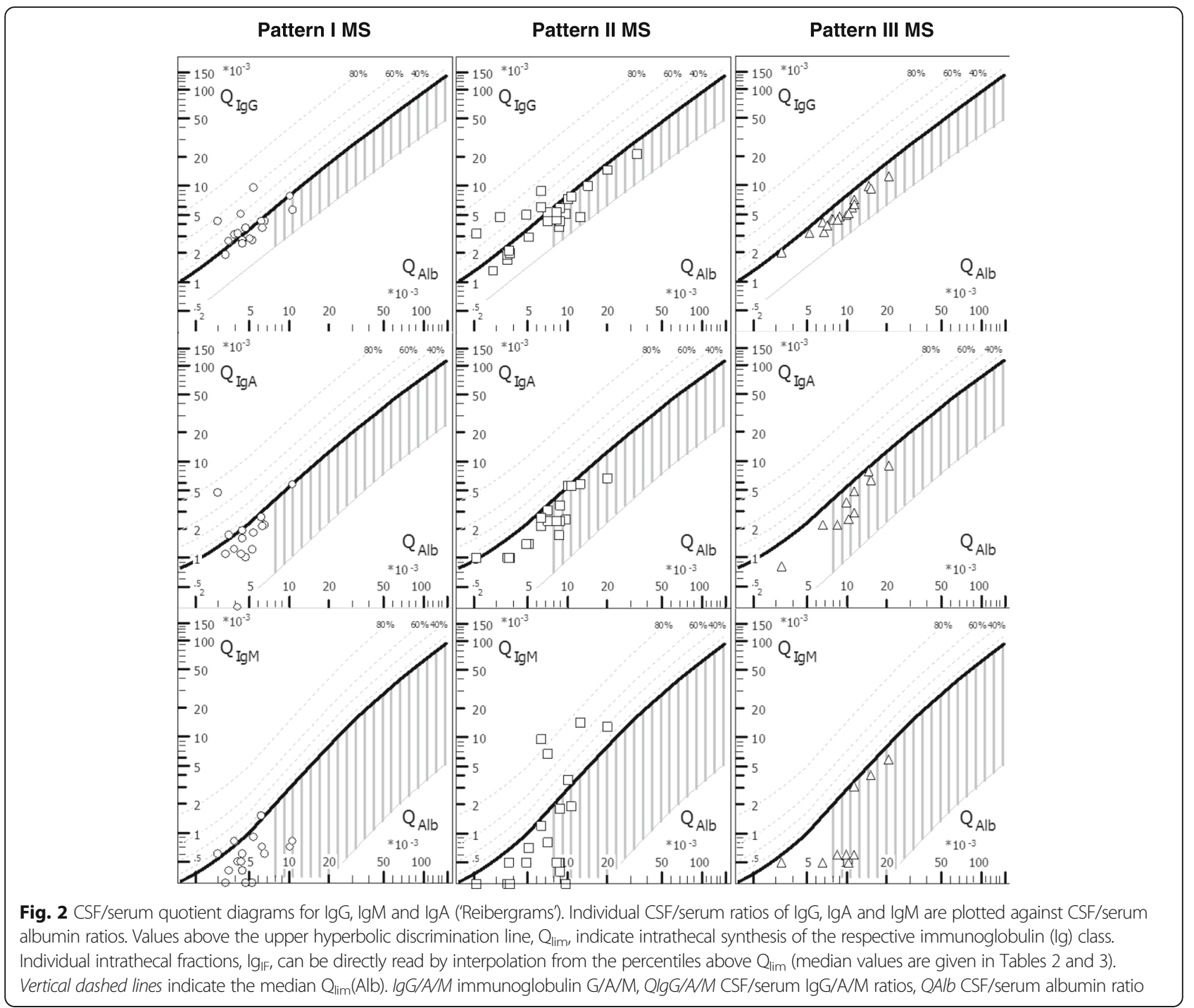

640, range 260-1277). TP CSF levels $>750 \mathrm{mg} / \mathrm{dl}$ were present in $8 / 43(18.6 \%)$ samples from patients with pattern II or III MS, but were not detected in any of the pattern I MS patients. As expected, TP CSF and QAlb were strongly correlated both in the total cohort and within each of the three subgroups $(P<0.0001)$.

\section{CSF L-lactate}

A trend towards higher median L-lactate level was noted in pattern II and III MS $(1.9 \mathrm{mmol} / \mathrm{L}$, range 0.8-3.3) compared with pattern I MS $(1.3 \mathrm{mmol} / \mathrm{L}$, range 1.1-2.35). Similarly, a higher proportion of patients with pattern II or III MS than patients with pattern I MS had elevated L-lactate levels at least once, but the difference was not statistically significant. See Table 8 for details.
Abnormal vs. normal results

Overall, 65/68 (95.6\%) LPs showed some abnormality (either intrathecal IgG, IgM or IgA synthesis; disturbed blood-CSF barrier function; or elevated WCC, TP or Llactate levels); the only three samples with normal results were all from patients with pattern II MS.

A summary of the statistically significant differences between groups can be found in Table 9 .

\section{Discussion}

In this study, we systematically analysed the results from 68 lumbar punctures in patients who were histopathologically diagnosed with pattern I, pattern II or pattern III MS according to previously published criteria [2]. The most striking finding was the lack of intrathecal IgG synthesis in the vast majority of samples from pattern II or pattern III MS patients. Intrathecal IgG synthesis is considered a diagnostic mainstay of MS. While previous 
Table 4 Immunoglobulin class response patterns

\begin{tabular}{cllllll}
\hline & Units & Pattern I MS & Pattern II + III MS & Pattern II MS & Pattern III MS & AQP4-IgG+ NMO [48] \\
\hline Three-class reaction & Samples & $0 / 16(0)$ & $0 / 32(0)$ & $0 / 21(0)$ & $0 / 11(0)$ & $0 / 87(0 \%)$ \\
Two-class reaction & Samples & $2 / 16(12.5)$ & $2 / 32(6.3)$ & $2 / 21(9.5)$ & $0 / 11(0)$ & $5 / 87(5.7 \%)$ \\
$\lg$ + IgM & Samples & 1 & 1 & 1 & 0 & $1 / 87(1.1 \%)$ \\
$\lg +\lg \mathrm{A}$ & Samples & 1 & 1 & 1 & 0 & $1 / 87(1.1 \%)$ \\
$\lg \mathrm{M}+\lg \mathrm{A}$ & Samples & 0 & 0 & 0 & 0 & $3 / 87(3.4 \%)$ \\
One-class reaction & Samples & $5 / 16(31.3)$ & $6 / 32(18.8)$ & $6 / 21(28.6)$ & $0 / 11(0)$ & $13 / 87(14.9 \%)$ \\
Only lgG & Samples & 4 & 2 & 2 & 0 & $5 / 87(5.7 \%)$ \\
Only lgM & Samples & 1 & 4 & 4 & 0 & $7 / 87(8 \%)$ \\
Only lgA & Samples & 0 & 0 & 0 & 0 & $1 / 87(1.1 \%)$ \\
\hline
\end{tabular}

Concentrations, ratios and fractions are reported as medians; range and total sample numbers examined are given in brackets $\mathrm{QlgG} / \mathrm{A} / \mathrm{M}=\mathrm{CSF} /$ serum $\mathrm{lgG} / \mathrm{A} / \mathrm{M}$ ratios

studies reported a frequency of OCB in MS of $90-98 \%$ in European patients, $73 \%$ of samples from pattern II or pattern III MS patients, including all pattern III patients, were negative in the present European cohort. Moreover, OCBs were positive only transiently in the only two patients with non-pattern I lesions and follow-up data. Transient OCBs have been reported in other neuroinflammatory diseases such as neuromyelitis optica (NMO), acute disseminated encephalomyelitis or CNS infection, whereas OCBs are thought to persist over the entire disease course of MS [24, 25]. Similarly, the polyspecific, intrathecal antiviral IgG response as defined by at least two elevated CSF/serum antibody indices to measles, rubella and/or (so-called MRZ reaction) varicella zoster virus, which is seen in around $70 \%$ of patients with MS (and considered to represent non-specific bystander B-cell activation) [10-14, 23], was missing in all patients with pattern II or III lesions tested. Some $91 \%$ of the pattern II and III MS samples did not even show a monospecific IgG response to one of the constituents of the
MRZR; in contrast, a reaction to at least one of the three viral agents has been found in $89-94 \%$ of MS patients [14, 19]. Together, this strongly suggests that patients with pattern II and pattern III lesions are immunopathophysiologically distinct from patients with 'pattern I MS' as well as from previous MS cohorts $[8,10,19]$.

This notion is further supported by the finding of additional, significant differences in CSF profiles between patients with pattern I lesions and patients with pattern II or III lesions. The latter group significantly more frequently had signs of compromised blood-CSF barrier function, had significantly higher CSF albumin concentrations and significantly higher and more frequently elevated CSF total protein levels (Tables 6 and 9; Fig. 3). In addition, an albuminocytological dissociation was more commonly seen in patients with type II or III lesions.

Although intraindividual persistence of a single lesion type over time has been shown in a larger cohort of biopsied patients $[4,5]$, there is an ongoing discussion

Table 5 Frequency of intrathecal lgG synthesis to infectious agents

\begin{tabular}{|c|c|c|c|c|c|c|c|c|}
\hline & Units & $\begin{array}{l}\text { MS, according to } \\
\text { literature [14] }\end{array}$ & $\begin{array}{l}\text { Pattern } \\
\text { I MS }\end{array}$ & $\begin{array}{l}\text { Pattern II } \\
+ \text { III MS }\end{array}$ & $\begin{array}{l}\text { Pattern } \\
\text { II MS }\end{array}$ & $\begin{array}{l}\text { Pattern } \\
\text { III MS }\end{array}$ & $\begin{array}{l}\text { AQP4-lgG+ NMO } \\
{[13,23,43,55,56]}\end{array}$ & $\begin{array}{l}\text { MOG-lgG+ } \\
\text { EM [35] }\end{array}$ \\
\hline \multirow[t]{2}{*}{ MRZ reaction $(\geq 2$ Als $>1.5)$} & Patients & $72 / 100(72)$ & $1 / 2(50)$ & $0 / 10(0)$ & 0/7 (0) & $0 / 3(0)$ & $1 / 42(2.4)$ & $0 / 11(0)$ \\
\hline & Samples & $72 / 100(72)$ & $1 / 2(50)$ & $0 / 12(0)$ & $0 / 8(0)$ & $0 / 4(0)$ & $1 / 42(2.4)$ & $0 / 11(0)$ \\
\hline $\mathrm{M}$ and/or $\mathrm{R}$ and/or $\mathrm{Z}>1.5$ & Samples & 158/177 (89) & $1 / 2(50)$ & $1 / 11(9.1)$ & $0 / 8(0)$ & $1 / 3(33.3)$ & n.d. & n.d. \\
\hline Measles virus $\mathrm{Al}>1.5$ & Samples & 138/177 (78) & $1 / 2(50)$ & $1 / 10(10)$ & 0/8 (0) & $1 / 4(25)$ & $1 / 42(2.4)$ & $0 / 11(0)$ \\
\hline Rubella virus $\mathrm{Al}>1.5$ & Samples & $106 / 177(60)$ & $1 / 2(50)$ & $0 / 9(0)$ & $0 / 8(0)$ & $0 / 4(0)$ & $0 / 42(0)$ & $0 / 11(0)$ \\
\hline Zoster virus $\mathrm{Al}>1.5$ & Samples & $97 / 177(55)$ & $0 / 2(0)$ & $0 / 9(0)$ & $0 / 8(0)$ & $0 / 4(0)$ & $1 / 42(2.4)$ & $0 / 11(0)$ \\
\hline Herpes simplex virus Al >1.5 & Samples & 26/94 (28) & n.d. & $0 / 4(0)$ & $0 / 4(0)$ & $0 / 1(0)$ & n.d. & n.d. \\
\hline Epstein-Barr virus Al >1.5 & Samples & n.d. & $0 / 2(0)$ & $0 / 2(0)$ & n.d. & n.d. & n.d. & $0 / 2(0)$ \\
\hline Borrelia burgdorferi Al >1.5 & Samples & $0 / 1(0)$ & $0 / 3(0)$ & $0 / 6(0)$ & $0 / 2(0)$ & n.d. & $0 / 1(0)$ & $0 / 3(0)$ \\
\hline Toxoplasma gondii $\mathrm{Al}>1.5$ & Samples & n.d. & $0 / 1(0)$ & $0 / 1(0)$ & n.d. & n.d. & n.d. & $0 / 1(0)$ \\
\hline TPHA Al >1.5 & Samples & n.d. & 0/1 (0) & 0/1 (0) & n.d. & n.d. & n.d. & $0 / 1(0)$ \\
\hline
\end{tabular}

n.a. not applicable $A$ l antibody index, $M$ measles virus, $R$ rubella virus, TPHA Treponema pallidum haemagglutination assay, $Z$ varicella zoster virus 
Table 6 Blood-CSF barrier function and albumin levels

\begin{tabular}{|c|c|c|c|c|c|c|}
\hline & Units & Pattern I MS & Pattern II + III MS & Pattern II MS & Pattern III MS & AQP4-lgG+ NMO [48] \\
\hline $\mathrm{QAlb}>\mathrm{Q}_{\lim }(\mathrm{Alb})$ & Patients & $4 / 10(40)$ & $15 / 22(68.2)$ & $9 / 15(60)$ & $6 / 7(85.7)$ & n.d. \\
\hline QAlb > Q $\lim (A l b)$ & Samples & $4 / 17(23.5)$ & $30 / 43(69.8)$ & $16 / 26(61.5)$ & $14 / 17(82.4)$ & 75/147 (51\%) \\
\hline QAlb, all LPs & - & $4.81(3.01-10.8 ; 17)$ & $8.39(2.1-33.9 ; 42)$ & $7.3(2.1-33.9 ; 25)$ & $9(3.3-20.7 ; 17)$ & $7(2.3-57.1 ; 137)$ \\
\hline QAlb, QAlb pos. & - & $8.53(6.3-10.8 ; 4)$ & $9.8(6.4-33.9 ; 29)$ & $9(6.4-33.9 ; 15)$ & $10.25(6.8--20.7 ; 14)$ & $11.8(5.63-57.14 ; 70)$ \\
\hline Albumin CSF, all LPS & $\mathrm{mg} / \mathrm{L}$ & $204(95-517 ; 17)$ & $331(81.2-726 ; 41)$ & $255.5(81.2-599 ; 24)$ & $368(137-726 ; 17)$ & 284 (83.6-1890; 139) \\
\hline Albumin CSF, QAlb pos. & $\mathrm{mg} / \mathrm{L}$ & $366(251-517 ; 4)$ & $371.5(227-726 ; 28)$ & $312(227-599 ; 14)$ & $431(282-726 ; 14)$ & 437 (219-1890) \\
\hline Albumin serum, all LPS & $g / L$ & $40.6(29.3-49.5 ; 17)$ & $40(22.6-51.8 ; 41)$ & $39.85(22.6-51.4 ; 24)$ & $41.2(32.7-51.8 ; 17)$ & $40.7(19.7-67.9 ; 133)$ \\
\hline Albumin serum, QAlb pos. & $g / L$ & $43.15(37.7-47.9 ; 4)$ & $39.5(22.6-50.9 ; 27)$ & $35.55(22.6-46.8 ; 14)$ & $39.9(32.7-50.9 ; 13)$ & $39(19.7-55.9)$ \\
\hline \multicolumn{7}{|c|}{ Combined intrathecal IgG synthesis and disturbed blood-CSF barrier function } \\
\hline Patients, at least once & $N(\%)$ & $3 / 10(30)$ & $4 / 20(20)$ & $3 / 13(23.1)$ & $1 / 7(14.3)$ & n.d. \\
\hline All LPS & $N(\%)$ & $3 / 17(17.6)$ & $5 / 43(11.6)$ & $4 / 26(15.4)$ & $1 / 17(5.9)$ & $13 / 74(17.6 \%)$ \\
\hline
\end{tabular}

n.d. no data

$\mathrm{QAlb}=\mathrm{CSF} /$ serum albumin ratio. Concentrations and ratios are reported as medians; range and total sample numbers examined are given in brackets

on whether the various histopathological lesion patterns identified in MS really represent different entities or rather different stages of the same disease. Given that OCBs are a constant feature in MS, which once acquired does not normally vanish $[24,25]$ (except in rare patients treated with natalizumab [26, 27], a drug not used in any of our patients), the lack of OCB positivity or persistence in most pattern II and III patients provides particularly strong evidence in favour of the former hypothesis.

The term 'multiple sclerosis' refers to a clinicoradiologically defined syndrome. There is currently no proof that all patients presenting with acute CNS demyelination and dissemination in time and space share the same underlying pathogenesis. Instead, several lines of evidence suggest that the current 'phenotypic' definition of MS may cover more than one disease: (i) pathological studies have demonstrated histopathological heterogeneity among patients with MS [1, 4, 5]; (ii) clinical studies have shown differences in clinical presentation ('spinal MS'), course (primary progressive MS without relapses vs. relapsing-remitting MS), severity and prognosis ('benign MS'); (iii) MRI studies have suggested different lesion types in MS; and (iv) treatment trials have found 'nonresponders', i.e. patients in whom drugs shown to be effective in the majority of patients with MS were of no benefit. In accordance with that hypothesis, numerous patients previously diagnosed as having variants of MS or 'opticospinal MS' based on the current clinicoradiological consensus criteria were found over the past decade to have newly discovered humorally mediated diseases, pathophysiologically distinct from MS, that are now termed AQP4-IgG-positive 'neuromyelitis optica spectrum' disorder (NMOSD) [28-33] and MOG-IgG-positive encephalomyelitis (EM) [17, 34-42]. Of note, many of patients with AQP4-IgG-positive NMO or MOG-IgGpositive EM had previously been wrongly diagnosed with MS due to a significant overlap in clinical presentation and clinical criteria and, in consequence, had been wrongly treated with drugs approved for MS but not for NMO or MOG-EM [17, 34-36, 43-47]. In a large European cohort, more than $40 \%$ of AQP4-IgG-positive patients with NMOSD had been previously misdiagnosed with MS [43]. Similarly, McDonald's clinicoradiological criteria or Barkhofs's radiological for MS were met by 33 and 15\%, respectively, of all MOG-IgG-positive

Table 7 CSF white cell counts

\begin{tabular}{|c|c|c|c|c|c|c|}
\hline & Units & Pattern I MS & Pattern II + III MS & Pattern II MS & Pattern III MS & AQP4-lgG+ NMO [48] \\
\hline Pleocytosis & Samples & $5 / 13(38.5)$ & $16 / 40(40)$ & $6 / 26(23.1)$ & 10/14 (71.4) & 98/194 (50.5) \\
\hline WCC, all LPs & Cells/uL & $5(1-23 ; 13)$ & $3(0-267 ; 40)$ & $2(0-267 ; 26)$ & $8.5(3-24 ; 14)$ & $6(0-380 ; 182)$ \\
\hline WCC, if elevated & 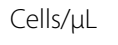 & $11(6-23 ; 5)$ & $14.5(6-267 ; 16)$ & $40(6-267 ; 6)$ & $10.5(7-24 ; 10)$ & $19(6-380 ; 98)$ \\
\hline WCC, $>40$ cells $/ \mu \mathrm{L}$ & Samples & 0/13 (0) & 3/36 (8.3) & $3 / 22(13.6)$ & $0 / 14(0)$ & 28/194 (14.4) \\
\hline WCC, $>40$ cells $/ \mu \mathrm{L}$ & 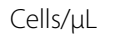 & n.a. & $69(55-267 ; 3)$ & $69(55-267 ; 3)$ & n.a. & $94(43-380 ; 28)$ \\
\hline $\begin{array}{l}\text { Albuminocytological } \\
\text { dissociation }\end{array}$ & Samples & $1 / 8(12.5)$ & 13/21 (61.9) & 10/18 (55.6) & $3 / 3(100)$ & 27/75 (33.3) \\
\hline
\end{tabular}

n.a. not applicable

WCC $=$ white cell count

WCC in the various groups are reported as medians; range and total sample numbers examined are given in brackets 
Table 8 CSF total protein and CSF L-lactate

\begin{tabular}{lllllll}
\hline & Units & Pattern I MS & Pattern II + III MS & Pattern II MS & Pattern III MS & AQP4-IgG+ NMO [48] \\
\hline CSF TP elevated & Patients & $3 / 9(33.3)$ & $14 / 20(70)$ & $8 / 13(61.5)$ & $6 / 7(85.7)$ & n.d. \\
CSF TP elevated & Samples & $3 / 15(20)$ & $29 / 43(67.4)$ & $15 / 26(57.7)$ & $14 / 17(82.4)$ & $80 / 152(52.6)$ \\
CSF TP, all LPS & $\mathrm{mg} / \mathrm{L}$ & $375(187-750 ; 15)$ & $550(168-1930 ; 43)$ & $501(168-1930 ; 26)$ & $640(260-1277 ; 17)$ & $473(198-3620 ; 147)$ \\
CSF TP, if elevated & $\mathrm{mg} / \mathrm{L}$ & $724(532-750 ; 3)$ & $622(470-1930 ; 29)$ & $571(471-1930 ; 15)$ & $693(470-1277 ; 14)$ & $780(45.4-3620 ; 68)$ \\
CSF TP, $>750 \mathrm{mg} / \mathrm{L}$ & $\mathrm{mg} / \mathrm{L}$ & $0 / 15(0)$ & $8 / 43(18.6)$ & $4 / 26(15.4)$ & $4 / 17(23.5)$ & $41 / 147(28)$ \\
CSF lactate elevated & Patients & $2 / 8(25)$ & $6 / 14(42.9)$ & $5 / 11(45.5)$ & $1 / 3(33.3)$ & n.d. \\
CSF lactate elevated & Samples & $2 / 9(22.2)$ & $7 / 28(25)$ & $6 / 20(30)$ & $1 / 8(12.5)$ & $27 / 83(32.5)$ \\
CSF lactate, all LPs & $\mathrm{mmol} / \mathrm{L}$ & $1.3(1.1-2.35 ; 11)$ & $1.9(0.8-3.3 ; 28)$ & $1.85(0.8-3.3 ; 20)$ & $1.9(1.36-2.9 ; 8)$ & $1.97(0.87-6.8 ; 80)$ \\
CSF lactate, if elevated & $\mathrm{mmol} / \mathrm{L}$ & $2.175(2-2.35 ; 2)$ & $2.5(2.2-3.3 ; 7)$ & $2.4(2.2-3.3 ; 6)$ & $2.9(2.9-2.9 ; 1)$ & $2.9(2.1-6.8 ; 27)$ \\
CSF lactate, $>3$ mmol/L & $\mathrm{mmol} / \mathrm{L}$ & $0 / 11(0)$ & $1 / 28(3.6)$ & $1 / 20(5)$ & $0 / 8(0)$ & $27 / 80(33.8)$ \\
\hline
\end{tabular}

n.d. no data

Concentrations are reported as medians; range and total sample numbers examined are given in brackets

patients in a recent study [35]. Wrong diagnosis and, accordingly, false treatment for MS may have caused disease exacerbation and worse outcome in some of those patients, underlining the clinical importance of studies investigating potential heterogeneity in MS [45-47].

AQP4-IgG-positive NMO lesions, MOG-IgG-positive EM lesions and pattern II MS lesions share important histopathological similarities in that all three are characterized by antibody and complement deposits. It is therefore of particular note that many of the CSF findings from patients with pattern II lesions were more similar to what has been reported in AQP4-IgGpositive NMO [43, 48, 49] and MOG-IgG-positive EM $[17,34-36]$ than to what was found in patients with pattern I lesions in our study (Tables 1, 2, 3, 4, 5, 6, 7, and 8). However, the majority of patients with pattern II and pattern III lesions are negative for AQP4-IgG and MOG-IgG, as were all 10 patients with pattern II or III lesions and available serum samples in the present study $[18,50,51]$, suggesting a potential role of other, so far unknown autoantibodies in this condition. In contrast, the CSF findings associated with pattern I lesions were much more in line with what one would expect in patients with typical MS.

Of interest, AQP4-IgG-positive NMO is characterized by a marked female predominance (male to female ratio 1:10-12) [43]; in contrast, the male to female ratio was 1:2.2 in the pattern II patients and thus more similar to what has been reported in MS and in MOG-IgGpositive EM [35].

In Germany, $\geq 95 \%$ of all MS patients are positive for CSF-restricted OCB $[8,10,19]$. The fact that most pattern II and III patients had no CSF-restricted OCB in our study therefore implies that pattern II and III MS cannot account for the majority of patients with MS. With most pattern II and III patients belonging to the small subgroup of $<5 \%$ of OCB-negative MS cases, pattern II MS and pattern III MS are probably rare conditions.

This seems to be in contrast to histopathological studies that reported a lower proportion of pattern I cases. However, there are several reasons why pattern II and III cases may be overrepresented in neuropathological cohorts:

(a) As OCBs are considered a hallmark of MS, it seems reasonable to conceive that patients with clinical and radiological findings suggestive of MS but no OCB are more likely to undergo brain biopsy or autopsy than those with OCB. The lack of intrathecal IgG synthesis in patients with pattern II or pattern III lesions may thus result in overrepresentation of pattern II and pattern III patients in histopathological studies.

(b) Tumefactive lesions are more commonly found in pattern II and III patients and certainly tend to prompt biopsies and autopsies more often than conventional lesions. Similarly, most pattern II and III patients in autopsy studies had died from highly active disease (in particular, patients with pattern III lesions, who often had died from fulminant disease within a few months from onset). However, fulminant and highly active disease definitely also tend to prompt biopsy and autopsy. This may again result in an overrepresentation of pattern II/III patients in biopsy/autopsy studies.

(c) Some patients with pattern II lesions are positive for MOG-IgG [4, 18, 52]. MOG encephalomyelitis shows a strong clinical and paraclinical overlap with MS but also significant differences such as ADEM-like presentation, longitudinally extensive and bilateral $\mathrm{ON}$, longitudinally extensive myelitis [17, 34-36]. Again, such 'MS-atypical' presentations may tend to prompt 
Table 9 Summary of differences in CSF parameters between various MS subgroups as observed in the present study

\begin{tabular}{|c|c|c|c|}
\hline Parameter & Diagnostic groups & Results & $P$ values \\
\hline \multirow[t]{7}{*}{ CSF-restricted OCB, all LPS } & Pattern I & 15/17 (88.2) & $P<0.00004$ \\
\hline & Patterns II + III & 10/37 (27) & \\
\hline & Reiber et al. [19] & $262 / 267(98)$ & $P<0.000001$ \\
\hline & Patterns II + III & 10/37 (27) & \\
\hline & Pattern I & 15/17 (88.2) & $P<0.0001$ \\
\hline & Pattern II & $9 / 25(36)$ & \\
\hline & Pattern III & $1 / 12(8.3)$ & \\
\hline \multirow[t]{4}{*}{ CSF-restricted OCB, patients } & Pattern I & $8 / 10(80)$ & $P<0.03$ \\
\hline & Patterns II + III & $7 / 22(31.8)$ & \\
\hline & Reiber et al. [19] & $262 / 267(98)$ & $P<0.000001$ \\
\hline & Patterns II + III & $7 / 22(31.8)$ & \\
\hline \multirow[t]{4}{*}{ CSF-restricted OCB, patients, w/o transient OCBs } & Pattern I & $8 / 10(80)$ & $P<0.006$ \\
\hline & Patterns II + III & $5 / 22(22.7)$ & \\
\hline & Reiber et al. [19] & $262 / 267(98)$ & $P<0.00001$ \\
\hline & Patterns II + III & $5 / 22(22.7)$ & \\
\hline \multirow[t]{2}{*}{ MRZ reaction ( $\geq 2$ Als $>1.5)$, all LPs } & Jarius et al. [23] & $397 / 546(69)$ & $P<0.000001$ \\
\hline & Patterns II + III & $0 / 12(0)$ & \\
\hline \multirow[t]{2}{*}{$M$ and/or $R$ and/or $Z>1.5$, all LPs } & Reiber et al. [19] & 158/177 (89) & $P<0.000001$ \\
\hline & Patterns II + III & $0 / 12(0)$ & \\
\hline \multirow[t]{2}{*}{ QlgG $>Q_{\lim }(\lg G)$, all LPs } & Pattern I & $7 / 17(41.2)$ & $P<0.03$ \\
\hline & Patterns II + III & $5 / 41(12.2)$ & \\
\hline \multirow[t]{5}{*}{ QAlb $>Q_{\text {lim }}($ Alb), all LPs } & Pattern I & $4 / 17(23.5)$ & $P<0.002$ \\
\hline & Patterns II + III & $30 / 43(69.8)$ & \\
\hline & Pattern I & $4 / 17(23.5)$ & $P=0.002$ \\
\hline & Pattern ॥ & $16 / 26(61.5)$ & \\
\hline & Pattern III & $14 / 17(82.4)$ & \\
\hline \multirow[t]{2}{*}{ QAlb, all LPs } & Pattern I & $4.81(3.01-10.8 ; 17)$ & $P<0.005$ \\
\hline & Patterns II + III & $8.39(2.1-33.9 ; 42)$ & \\
\hline \multirow[t]{2}{*}{ Albumin CSF, all LPs } & Pattern I & $204(95-517 ; 17)$ & $P<0.009$ \\
\hline & Patterns II + III & $331(81.2-726 ; 41)$ & \\
\hline \multirow[t]{2}{*}{ Total CSF protein elevated, all LPS } & Pattern I & $3 / 15(20)$ & $P<0.003$ \\
\hline & Patterns II + III & 29/43 (67.4) & \\
\hline \multirow[t]{2}{*}{ Total CSF protein, all LPS } & Pattern I & $375(187-750 ; 15)$ & $P<0.007$ \\
\hline & Patterns II + III & $550(168-1930 ; 43)$ & \\
\hline \multirow[t]{2}{*}{ Albuminocytological dissociation, all LPs } & Pattern I & $1 / 8(12.5)$ & $P<0.04$ \\
\hline & Patterns $\|+\| I$ & $13 / 21(61.9)$ & \\
\hline
\end{tabular}




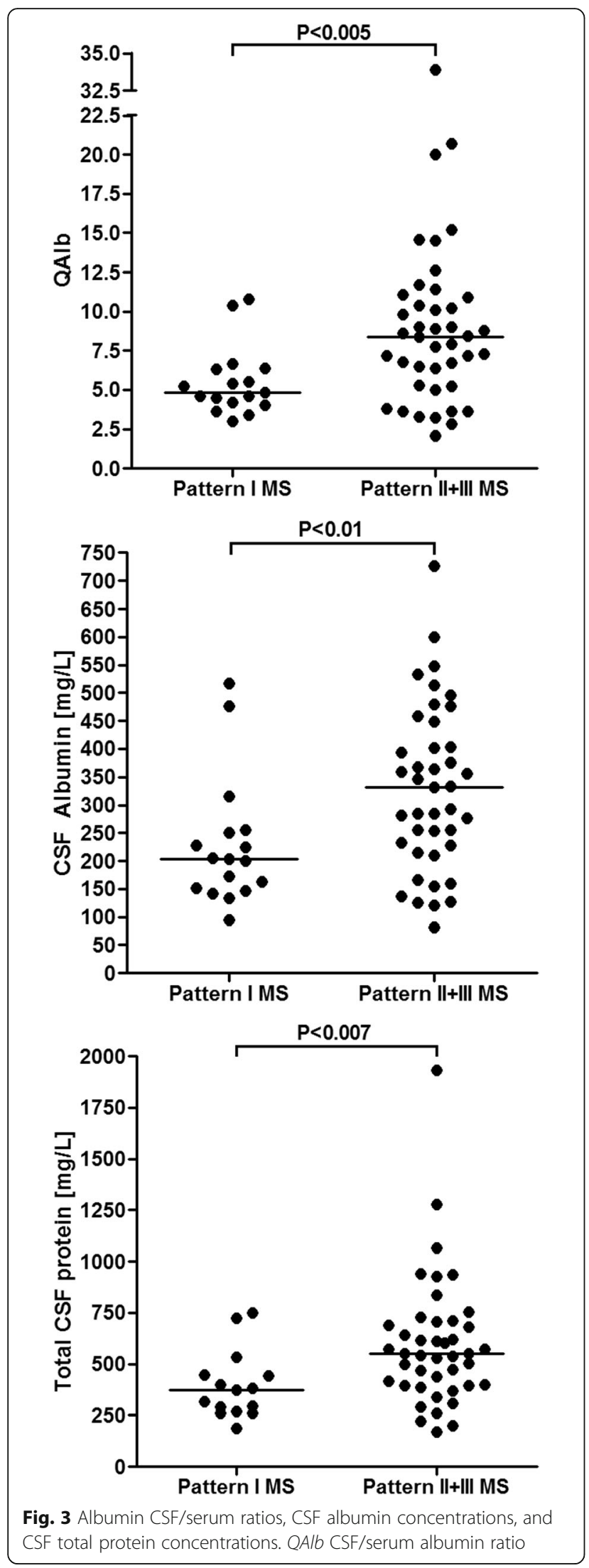

biopsy or autopsy and, thus, artificially increase the proportion of pattern II cases in biopsy/autopsy studies.

Our results indicate that the proportion of patients with type II and type III lesions may be particularly high among OCB-negative patients diagnosed with MS. While only $2-10 \%$ of patients with MS are negative for $\mathrm{OCB}$ according to the literature, this subset is not small in absolute numbers, given the high prevalence of MS in some Western populations (e.g. 2.3 million patients worldwide; $~ 140,000$ patients in Germany). Given that fact and considering that differences in pathophysiology may well translate into different treatment requirements, as already shown in AQP4-IgG-positive NMO [47] and suggested for MOG-IgG-positive EM [34, 35], studies aiming at enhancing our understanding of the immunopathophysiology of pattern II and III lesions seem highly warranted.

Importantly, the frequency of OCB has been reported to increase with latitude [53,54]. Whether this implies a higher proportion of patients with 'pattern I' lesions at higher latitudes is currently unknown but deserves to be addressed in future studies.

We acknowledge that our study has both strengths and limitations. While we count the high number of CSF samples included $(n=68)$ and the high number of CSF parameters analysed among the strengths of our study, the retrospective and exploratory study design is a potential limitation. However, prospective studies would be extremely difficult or even impossible to perform given that brain biopsies are only very rarely performed in patients with MS and that lumbar puncture is not anymore required to make the diagnosis of MS according to the current international diagnostic criteria [3].

\section{Conclusions}

In summary, the present study provides additional, strong evidence for pattern I MS on the one hand and pattern II MS and pattern III MS on the other hand being distinct entities by demonstrating significant differences in CSF findings between patients with pattern I lesions and patients with pattern II or pattern III lesions, especially with regard to intrathecal IgG synthesis. Of particular note, the CSF profiles present in the pattern II/III subgroup were more similar to those reported in AQP4-IgG-positive NMO and MOG-IgGpositive EM than to those classically considered typical for MS. Further studies are now warranted to confirm our findings in larger, international cohorts. Such studies need to take into account the recently reported increase in frequency of CSF oligoclonal banding in MS with latitude [53, 54]. 


\section{Abbreviations}

AQP4: Aquaporin-4; CNS: Central nervous system; CSF: Cerebrospinal fluid; EM: Encephalomyelitis; IgA: Immunoglobulin A; IgG: Immunoglobulin G; IgM: Immunoglobulin M; MOG: Myelin oligodendrocyte glycoprotein; MRZR: Measles, rubella, zoster reaction; MS: Multiple sclerosis; NMO: Neuromyelitis optica; NMOSD: NMO spectrum disorder; OCBs: Oligoclonal bands; QAlb: Albumin CSF/serum ratio; QlgG/A/M: IgG/A/M CSF/serum ratio; TP: Total protein; TPHA: Treponema pallidum haemagglutination assay; WCC: White cell count

\section{Acknowledgements}

This work was supported by research grants from the Dietmar Hopp Stiftung to BW, from Merck Serono to BW, from the German Federal Ministry of Education and research-funded Competence Network Multiple Sclerosis (KKNMS) to BW, WB and IM, and by a research fellowship from the European Committee for Treatment and Research in Multiple Sclerosis (ECTRIMS) to SJ. The authors are thankful to Mrs. Anna Eschlbeck and to the Nikon Imaging Center for the excellent technical assistance. We acknowledge the financial support by Deutsche Forschungsgemeinschaft and Ruprecht-Karls-Universität Heidelberg within the funding programme Open Access Publishing.

\section{Funding}

The work of BW was supported by research grants from the Dietmar Hopp Stiftung and from Merck Serono. The work of BW, WB and IM was supported by the German Federal Ministry of Education and Research (BMBF/KKNMS, Competence Network Multiple Sclerosis). The work of SJ was supported by a research grant from the European Committee for Treatment and Research in Multiple Sclerosis (ECTRIMS).

\section{Availability of data and materials}

The datasets generated during and/or analysed during the current study are not publicly available due to local data protection requirements but are available from the corresponding author on reasonable request in an anonymized fashion.

\section{Authors' contributions}

SJ conceived and designed the study, analysed the CSF data and wrote the manuscript. WB, FK and IM provided the histopathological data. WB, FK, IM, BW, FP and KR provided CSF and clinical data. All authors were involved in revising the manuscript for intellectual content. All authors read and approved the final draft before submission.

\section{Ethics approval and consent to participate}

The study was approved by the institutional review board of the University of Göttingen, and the patients gave written informed consent.

\section{Consent for publication}

Not applicable.

\section{Competing interests}

BW has received research grants, speaking fees and travel grants from Merck Serono, Biogen, Teva, Novartis, Sanofi Genzyme, Bayer Healthcare, Biotest and the Dietmar Hopp Foundation. KR has received research support from Novartis as well as speaking fees and travel grants from Guthy Jackson Charitable Foundation, Bayer Healthcare, Biogen Idec, Merck Serono, Sanofi Genzyme, Teva, Roche, and Novartis, none of which is related to the present study. FrP has received research support from Bayer, Novartis, Biogen Idec, Teva, Sanofi-Aventis/Genzyme, Merck Serono, Alexion, Chugai, Arthur Arnstein Stifung Berlin, Guthy Jackson Charitable Foundation and the US National Multiple Sclerosis Society; has received travel funding and/or speaker honoraria from Bayer, Novartis, Biogen Idec, Teva, Sanofi Aventis/Genzyme and Merck Serono; and has consulted for Sanofi Genzyme, Biogen Idec, and Medlmmune; none of which is related to the present paper. The other authors report no competing interests. WB has received honoraria for lectures by Bayer Vital, Biogen, Merck Serono, Teva, Genzyme, Roche and Novartis. He is a member of scientific advisory boards for Teva, Biogen, Novartis and Genzyme. WB receives research support from Teva, Biogen, Genzyme and Novartis.

\section{Publisher's Note}

Springer Nature remains neutral with regard to jurisdictional claims in published maps and institutional affiliations.

\section{Author details}

${ }^{1}$ Molecular Neuroimmunology Group, Department of Neurology, University of Heidelberg, Heidelberg, Germany. '2Department of Neuropathology, University of Göttingen, Göttingen, Germany. ${ }^{3}$ Department of Neurology, Charité University Medicine Berlin, Berlin, Germany. ${ }^{4}$ NeuroCure Clinical Research Center and Clinical and Experimental Multiple Sclerosis Research Center, Berlin, Germany.

Received: 1 February 2017 Accepted: 26 July 2017

Published online: 29 August 2017

\section{References}

1. Lucchinetti CF, Bruck W, Lassmann H. Evidence for pathogenic heterogeneity in multiple sclerosis. Ann Neurol. 2004;56:308.

2. Lucchinetti C, Bruck W, Parisi J, Scheithauer B, Rodriguez M, Lassmann H. Heterogeneity of multiple sclerosis lesions: implications for the pathogenesis of demyelination. Ann Neurol. 2000;47:707-17.

3. Polman CH, Reingold SC, Banwell B, Clanet M, Cohen JA, Filippi M, Fujihara K, Havrdova E, Hutchinson M, Kappos L, et al. Diagnostic criteria for multiple sclerosis: 2010 revisions to the McDonald criteria. Ann Neurol. 2011;69:292-302

4. Konig FB, Wildemann B, Nessler S, Zhou D, Hemmer B, Metz I, Hartung HP, Kieseier BC, Bruck W. Persistence of immunopathological and radiological traits in multiple sclerosis. Arch Neurol. 2008;65:1527-32.

5. Metz I, Weigand SD, Popescu BF, Frischer JM, Parisi JE, Guo Y, Lassmann H, Bruck W, Lucchinetti CF. Pathologic heterogeneity persists in early active multiple sclerosis lesions. Ann Neurol. 2014;75:728-38.

6. Tumani H, Deisenhammer F, Giovannoni G, Gold R, Hartung HP, Hemmer B, Hohlfeld R, Otto M, Stangel M, Wildemann B, Zettl UK. Revised McDonald criteria: the persisting importance of cerebrospinal fluid analysis. Ann Neurol. 2011;70:520. author reply 521

7. Franciotta D, Columba-Cabezas S, Andreoni L, Ravaglia S, Jarius S, Romagnolo S, Tavazzi E, Bergamaschi R, Zardini E, Aloisi F, Marchioni E. Oligoclonal lgG band patterns in inflammatory demyelinating human and mouse diseases. J Neuroimmunol. 2008;200:125-8.

8. Reiber H, Teut M, Pohl D, Rostasy KM, Hanefeld F. Paediatric and adult multiple sclerosis: age-related differences and time course of the neuroimmunological response in cerebrospinal fluid. Mult Scler. 2009;15:1466-80.

9. Andersson M, Alvarez-Cermeno J, Bernardi G, Cogato I, Fredman P, Frederiksen J, Fredrikson S, Gallo P, Grimaldi LM, Gronning M, et al. Cerebrospinal fluid in the diagnosis of multiple sclerosis: a consensus report. J Neurol Neurosurg Psychiatry. 1994;57:897-902.

10. Reiber H. Cerebrospinal fluid-physiology, analysis and interpretation of protein patterns for diagnosis of neurological diseases. Mult Scler. 1998;4:99-107.

11. Jarius S, Eichhorn P, Jacobi C, Wildemann B, Wick M, Voltz R. The intrathecal, polyspecific antiviral immune response: specific for MS or a general marker of CNS autoimmunity? J Neurol Sci. 2009;280:98-100.

12. Jarius $S$, Eichhorn $P$, Wildemann B, Wick M. Usefulness of antibody index assessment in cerebrospinal fluid from patients negative for total-lgG oligoclonal bands. Fluids Barriers CNS. 2012;9:14.

13. Jarius $S$, Franciotta $D$, Bergamaschi R, Rauer $S$, Wandinger KP, Petereit HF, Maurer M, Tumani H, Vincent A, Eichhorn P, et al. Polyspecific, antiviral immune response distinguishes multiple sclerosis and neuromyelitis optica. J Neurol Neurosurg Psychiatry. 2008;79:1134-6.

14. Felgenhauer $\mathrm{K}$, Reiber $\mathrm{H}$. The diagnostic significance of antibody specificity indices in multiple sclerosis and herpes virus induced diseases of the nervous system. Clin Investig. 1992;70:28-37.

15. Jarius S, Probst C, Borowski K, Franciotta D, Wildemann B, Stoecker W, Wandinger KP. Standardized method for the detection of antibodies to aquaporin-4 based on a highly sensitive immunofluorescence assay employing recombinant target antigen. J Neurol Sci. 2010;291:52-6.

16. Jarius S, Paul F, Fechner K, Ruprecht K, Kleiter I, Franciotta D, Ringelstein M, Pache F, Aktas O, Wildemann B. Aquaporin-4 antibody testing: direct comparison of M1-AQP4-DNA-transfected cells with leaky scanning versus M23-AQP4-DNA-transfected cells as antigenic substrate. J Neuroinflammation. 2014;11:129.

17. Jarius S, Ruprecht K, Kleiter I, Borisow N, Asgari N, Pitarokoili K, Pache F, Stich O, Beume LA, Hummert MW, et al. MOG-lgG in NMO and related disorders: a multicenter study of 50 patients. Part 1: frequency, syndrome specificity, influence of disease activity, long-term course, association with AQP4-IgG, and origin. J Neuroinflammation. 2016;13:279. 
18. Jarius S, Metz I, Konig FB, Ruprecht K, Reindl M, Paul F, Bruck W, Wildemann B. Screening for MOG-lgG and 27 other anti-glial and anti-neuronal autoantibodies in 'pattern II multiple sclerosis' and brain biopsy findings in a MOG-lgG-positive case. Mult Scler. 2016;22:1541-9.

19. Reiber $\mathrm{H}$, Ungefehr $\mathrm{S}$, Jacobi $\mathrm{C}$. The intrathecal, polyspecific and oligoclonal immune response in multiple sclerosis. Mult Scler. 1998:4:111-7.

20. Reiber H. Flow rate of cerebrospinal fluid (CSF) - a concept common to normal blood-CSF barrier function and to dysfunction in neurological diseases. J Neurol Sci. 1994;122:189-203.

21. Petereit HF, Sindern E, Wick M. CSF diagnostics. Guidelines and catalogue of methods of the German Society for Cerebrospinal Fluid Diagnostics and Clinical Neurochemistry. Heidelberg: Springer; 2007.

22. Wildemann B, Oschmann P, Reiber H. Laboratory diagnosis in neurology Stuttgart, New York: Thieme; 2011.

23. Jarius S, Eichhorn P, Franciotta D, Petereit HF, Akman-Demir G, Wick M Wildemann $B$. The MRZ reaction as a highly specific marker of multiple sclerosis: re-evaluation and structured review of the literature. J Neurol. 2017;264:453-66.

24. Walsh MJ, Tourtellotte WW. Temporal invariance and clonal uniformity of brain and cerebrospinal lgG, IgA, and IgM in multiple sclerosis. J Exp Med. 1986;163:41-53.

25. Petereit HF, Reske D. Expansion of antibody reactivity in the cerebrospinal fluid of multiple sclerosis patients_-follow-up and clinical implications. Cerebrospinal Fluid Res. 2005;2:3.

26. von Glehn F, Farias AS, de Oliveira AC, Damasceno A, Longhini AL, Oliveira EC, Damasceno BP, Santos LM, Brandao CO. Disappearance of cerebrospinal fluid oligoclonal bands after natalizumab treatment of multiple sclerosis patients. Mult Scler. 2012;18:1038-41.

27. Harrer A, Tumani H, Niendorf S, Lauda F, Geis C, Weishaupt A, Kleinschnitz C, Rauer S, Kuhle J, Stangel M, et al. Cerebrospinal fluid parameters of B cellrelated activity in patients with active disease during natalizumab therapy. Mult Scler. 2013;19:1209-12.

28. Lennon VA, Kryzer TJ, Pittock SJ, Verkman AS, Hinson SR. IgG marker of optic-spinal multiple sclerosis binds to the aquaporin-4 water channel. J Exp Med. 2005;202:473-7.

29. Lennon VA, Wingerchuk DM, Kryzer TJ, Pittock SJ, Lucchinetti CF, Fujihara K, Nakashima I, Weinshenker BG. A serum autoantibody marker of neuromyelitis optica: distinction from multiple sclerosis. Lancet. 2004;364:2106-12.

30. Levy M, Wildemann B, Jarius S, Orellano B, Sasidharan S, Weber MS, Stuve O. Immunopathogenesis of neuromyelitis optica. Adv Immunol. 2014;121:213-42.

31. Jarius S, Paul F, Franciotta D, Waters P, Zipp F, Hohlfeld R, Vincent A Wildemann B. Mechanisms of disease: aquaporin-4 antibodies in neuromyelitis optica. Nat Clin Pract Neurol. 2008;4:202-14.

32. Jarius $\mathrm{S}$, Wildemann B. AQP4 antibodies in neuromyelitis optica: diagnostic and pathogenetic relevance. Nat Rev Neurol. 2010;6:383-92.

33. Jarius S, Wildemann B, Paul F. Neuromyelitis optica: clinical features, immunopathogenesis and treatment. Clin Exp Immunol. 2014;176:149-64.

34. Jarius S, Kleiter I, Ruprecht K, Asgari N, Pitarokoili K, Borisow N, Hummert MW, Trebst C, Pache F, Winkelmann A, et al. MOG-lgG in NMO and related disorders: a multicenter study of 50 patients. Part 3: brainstem involvement-frequency, presentation and outcome. J Neuroinflammation. 2016;13:281.

35. Jarius S, Ruprecht K, Kleiter I, Borisow N, Asgari N, Pitarokoili K, Pache F, Stich O, Beume LA, Hummert MW, et al. MOG-IgG in NMO and related disorders: a multicenter study of 50 patients. Part 2: epidemiology, clinical presentation, radiological and laboratory features, treatment responses, and long-term outcome. J Neuroinflammation. 2016;13:280

36. Pache F, Zimmermann H, Mikolajczak J, Schumacher S, Lacheta A, Oertel FC, Bellmann-Strobl J, Jarius S, Wildemann B, Reindl M, et al. MOG-lgG in NMO and related disorders: a multicenter study of 50 patients. Part 4: afferent visual system damage after optic neuritis in MOG-lgG-seropositive versus AQP4-IgG-seropositive patients. J Neuroinflammation. 2016;13:282.

37. Mader S, Gredler V, Schanda K, Rostasy K, Dujmovic I, Pfaller K, Lutterotti A, Jarius S, Di Pauli F, Kuenz B, et al. Complement activating antibodies to myelin oligodendrocyte glycoprotein in neuromyelitis optica and related disorders. J Neuroinflammation. 2011;8:184.

38. Jarius $\mathrm{S}$, Wildemann B. The history of neuromyelitis optica. J Neuroinflammation. 2013;10:8.

39. Brain WR. Critical review: disseminated sclerosis. QJM. 1930;23:343-91.

40. Weinshenker BG, Wingerchuk DM, Nakashima I, Fujihara K, Lennon VA. OSMS is NMO, but not MS: proven clinically and pathologically. Lancet Neurol. 2006:5:110-1.
41. Zamvil SS, Slavin AJ. Does MOG Ig-positive AQP4-seronegative opticospinal inflammatory disease justify a diagnosis of NMO spectrum disorder? Neurol Neuroimmunol Neuroinflamm. 2015;2:e62.

42. Reindl M, Jarius S, Rostasy K, Berger T. Myelin oligodendrocyte glycoprotein antibodies: How clinically useful are they? Curr Opin Neurol. 2017;30(3):295-301.

43. Jarius S, Ruprecht K, Wildemann B, Kuempfel T, Ringelstein M, Geis C, Kleiter I, Kleinschnitz C, Berthele A, Brettschneider J, et al. Contrasting disease patterns in seropositive and seronegative neuromyelitis optica: a multicentre study of 175 patients. J Neuroinflammation. 2012;9:14

44. Wildemann B, Jarius S, Schwarz A, Diem R, Viehöver A, Hähnel S, Reindl M, Korporal-Kuhnke M. Failure of alemtuzumab therapy to control MOG encephalomyelitis. Neurology. 2017;89(2):207-9.

45. Palace J, Leite MI, Nairne A, Vincent A. Interferon Beta treatment in neuromyelitis optica: increase in relapses and aquaporin 4 antibody titers. Arch Neurol. 2010;67:1016-7.

46. Uzawa A, Mori M, Hayakawa S, Masuda S, Kuwabara S. Different responses to interferon beta-1b treatment in patients with neuromyelitis optica and multiple sclerosis. Eur J Neurol. 2010;17:672-6.

47. Trebst C, Jarius S, Berthele A, Paul F, Schippling S, Wildemann B, Borisow N, Kleiter I, Aktas O, Kumpfel T. Update on the diagnosis and treatment of neuromyelitis optica: recommendations of the Neuromyelitis Optica study group (NEMOS). J Neurol. 2013;261:1-16.

48. Jarius S, Paul F, Franciotta D, Ruprecht K, Ringelstein M, Bergamaschi R, Rommer $\mathrm{P}$, Kleiter I, Stich $\mathrm{O}$, Reuss $\mathrm{R}$, et al. Cerebrospinal fluid findings in aquaporin-4 antibody positive neuromyelitis optica: results from 211 lumbar punctures. J Neurol Sci. 2011;306:82-90.

49. Jarius S, Franciotta D, Paul F, Ruprecht K, Bergamaschi R, Rommer PS, Reuss R, Probst C, Kristoferitsch W, Wandinger KP, Wildemann B. Cerebrospinal fluid antibodies to aquaporin-4 in neuromyelitis optica and related disorders: frequency, origin, and diagnostic relevance. J Neuroinflammation. 2010;7:52.

50. Kale N, Pittock SJ, Lennon VA, Thomsen K, Roemer S, McKeon A, Lucchinetti CF. Humoral pattern II multiple sclerosis pathology not associated with neuromyelitis Optica IgG. Arch Neurol. 2009;66:1298-9.

51. Hahn S, Trendelenburg G, Scharf M, Denno Y, Brakopp S, Teegen B, Probst C, Wandinger KP, Buttmann M, Haarmann A, Szabados F, Vom Dahl M, Kümpfel T, Eichhorn P, Gold H, Paul F, Jarius S, Melzer N, Stöcker W, Komorowski L. Identification of the flotillin-1/2 heterocomplex as a target of autoantibodies in bona fide multiple sclerosis. J Neuroinflammation. 2017; 14(1):123.

52. Spadaro M, Gerdes LA, Mayer MC, Ertl-Wagner B, Laurent S, Krumbholz M, Breithaupt C, Högen T, Straube A, Giese A, et al. Histopathology and clinical course of MOG-antibody-associated encephalomyelitis. Ann Clin Transl Neurol. 2015:2(3):295-301.

53. Dobson R, Ramagopalan S, Davis A, Giovannoni G. Cerebrospinal fluid oligoclonal bands in multiple sclerosis and clinically isolated syndromes: a meta-analysis of prevalence, prognosis and effect of latitude. J Neurol Neurosurg Psychiatry. 2013;84:909-14.

54. Lechner-Scott J, Spencer B, de Malmanche T, Attia J, Fitzgerald M, Trojano M, Grand'Maison F, Gomez JA, Izquierdo G, Duquette P, et al. The frequency of CSF oligoclonal banding in multiple sclerosis increases with latitude. Mult Scler. 2012;18:974-82.

55. Pohl D, Rostasy K, Jacobi C, Lange P, Nau R, Krone B, Hanefeld F. Intrathecal antibody production against Epstein-Barr and other neurotropic viruses in pediatric and adult onset multiple sclerosis. J Neurol. 2010;257:212-6.

56. Brecht I, Weissbrich B, Braun J, Toyka KV, Weishaupt A, Buttmann M. Intrathecal, polyspecific antiviral immune response in oligoclonal band negative multiple sclerosis. PLoS One. 2012;7:e40431. 